\title{
Transcutaneous versus Total Serum Bilirubin Measurements in Preterm Infants
}

\author{
Thivia Jegathesan $^{a, b}$ Douglas M. Campbell ${ }^{b, c}$ Joel G. Ray ${ }^{a, d, e}$ Vibhuti Shah ${ }^{c, e, f}$ \\ Howard Berger $^{d, e}$ Robin Z. Hayeems ${ }^{e, g}$ Michael Sgro ${ }^{a, b, c}$ for the NeoHBC \\ anstitute of Medical Sciences, Temerty Faculty of Medicine, University of Toronto, Toronto, ON, Canada; \\ bDepartment of Pediatrics, and Li Ka Shing Knowledge Institute, Unity Health Toronto at St. Michael's Hospital, \\ Toronto, ON, Canada; 'Department of Pediatrics, Division of Neonatology, University of Toronto, Toronto, ON, \\ Canada; ${ }^{\mathrm{d} D e p a r t m e n t}$ of Obstetrics and Gynecology, and Li Ka Shing Knowledge Institute, Unity Health Toronto \\ at St. Michael's Hospital, Toronto, ON, Canada; eInstitute of Health Policy, Management and Evaluation, University \\ of Toronto, Toronto, ON, Canada; fDepartment of Paediatrics, Sinai Health, Toronto, ON, Canada; ${ }^{9}$ Child Health \\ Evaluative Sciences, Hospital for Sick Children, Toronto, ON, Canada
}

\section{Keywords}

Hyperbilirubinemia $\cdot$ Newborn screening $\cdot$ Jaundice $\cdot$

Neonatal pain · Blood tests

\begin{abstract}
Introduction: Transcutaneous bilirubin (TcB) measurement offers a noninvasive approach for bilirubin screening; however, its accuracy in preterm infants is unclear. This study determined the agreement between TCB and total serum bilirubin (TSB) among preterm infants. Methods: A multisite prospective cohort study was conducted at 3 NICUs in Ontario, Canada, September 2016 to June 2018. Among 296 preterm infants born at $24^{0 / 7}$ to $35^{6 / 7}$ weeks, $856 \mathrm{TcB}$ levels were taken at the forehead, sternum, and before and after the initiation of phototherapy with TSB measurements. Bland-Altman plots and 95\% limits of agreement (LOA) expressed agreement between TCB and TSB. Results: The overall mean TcB-TSB difference was $-24.5 \mu \mathrm{mol} / \mathrm{L}(95 \% \mathrm{LOA}$ -103.3 to 54.3 ), $1.6 \mu \mathrm{mol} / \mathrm{L}$ (95\% LOA -73.4 to 76.5 ) before phototherapy, and $-31.1 \mu \mathrm{mol} / \mathrm{L}$ (95\% LOA -105.5 to 43.4 ) after the initiation of phototherapy. The overall mean TcB-
\end{abstract}

karger@karger.com www.karger.com/neo

Karger"

BOPEN ACCESS
(C) 2021 The Author(s)

Published by S. Karger AG, Basel

This is an Open Access article licensed under the Creative Commons Attribution-NonCommercial-4.0 International License (CC BY-NC) (http://www.karger.com/Services/OpenAccessLicense), applicable to the online version of the article only. Usage and distribution for commercial purposes requires written permission.
TSB difference was $-15.2 \mu \mathrm{mol} / \mathrm{L}$ ( $95 \% \mathrm{LOA}-86.8$ to 56.3 ) at the forehead and $-24.4 \mu \mathrm{mol} / \mathrm{L}$ ( $95 \% \mathrm{LOA}-112.9$ to 64.0 ) at the sternum. The mean TCB-TSB difference was $-31.4 \mu \mathrm{mol} / \mathrm{L}$ (95\% LOA -95.3 to 32.4 ) among infants born 24-28 weeks, $-25.5 \mu \mathrm{mol} / \mathrm{L}(95 \% \mathrm{LOA}-102.7$ to 51.8$)$ at $29-32$ weeks, and $-15.9 \mu \mathrm{mol} / \mathrm{L}(95 \% \mathrm{LOA}-107.4$ to 75.6$)$ at 33-35 weeks. Measures did not differ by maternal ethnicity. Conclusion: Among preterm infants, TCB may offer a noninvasive, immediate approach to screening for hyperbilirubinemia with more careful use in preterm infants born at $<33$ weeks' gestation, as TcB approaches treatment thresholds. Its underestimation of TSB after the initiation of phototherapy warrants the use of TSB for clinical decision-making after the initiation of phototherapy.

(c) 2021 The Author(s).

Published by S. Karger AG, Basel

\section{Introduction}

The decline in acute and chronic bilirubin encephalopathies in Canada and the USA can be attributed, in part, to the adoption of American Academy of Pediatrics' 
and Canadian Paediatric Society's recommendations to perform routine total serum bilirubin (TSB) screening in infants born at $>35$ weeks' gestation prior to hospital discharge [1-3] and among preterm infants born at 24-35 weeks' gestation $[4,5]$.

Although obtaining TSB is the most common way to measure bilirubin levels in infants, blood sampling can be painful and a time-consuming procedure $[6,7]$. Studies have also reported concerns about frequent TSB measurements, such as the increased risk of infection and anemia, particularly among extremely preterm infants $[8$, 9]. Furthermore, repeated procedural pain and stress have been documented in preterm infants [10].

In term and near-term infants, the use of noninvasive transcutaneous bilirubin $(\mathrm{TcB})$ measurement has gained use in clinical practice, as it can reduce the frequency of TSB tests when TSB concentrations are $<240 \mu \mathrm{mol} / \mathrm{L}(<14$ $\mathrm{mg} / \mathrm{dL})[1,2]$. There is a conflicting body of research on the use of TcB in preterm infants $\leq 35$ weeks' gestation. Prior studies included small sample sizes, with conflicting data reporting the effects of phototherapy and anatomical site of measurement [11-13]. There are also limited data reporting the effects of ethnicity on $\mathrm{TcB}$ in preterm infants $[13,14]$. In addition, few recent studies have stratified agreement between TcB and TSB by gestational age groups of prematurity among preterm infants, especially after the initiation of phototherapy [12].

The primary objective of this study was to evaluate the agreement (i.e., limits of agreement [LOA]) between $\mathrm{TcB}$ and TSB measurements among preterm infants born at $24^{0 / 7}$ to $35^{6 / 7}$ weeks' gestation. We also assessed the agreement between TcB and TSB before and after the initiation of phototherapy, by anatomical site of measurement and the infant's ethnicity.

\section{Materials and Methods}

A multisite prospective cohort study was completed in Ontario at St. Michael's Hospital, Sinai Health, and Hamilton Health Sciences Centre from September 2016 to June 2018. Eligible participants were preterm infants born at $24^{0 / 7}$ to $35^{6 / 7}$ weeks' gestation and admitted to participating sites. Excluded were those with a condition that could interfere with $\mathrm{TcB}$ measurements, such as hydrops fetalis, congenital malformation, diffuse cutaneous conditions, infection, or purpura.

The JM-105 TcB device (Drager Medical Systems Inc., Telford, Philadelphia) was used to measure $\mathrm{TcB}$ in each participating infant within 15 min of every TSB measurement. TSB samples were collected by venous or capillary blood sampling as per clinical decision and analyzed by spectrophotometry using Beckman Coulter's AU680 automated analyzer (online suppl. Text 1; for all online suppl. material, see www.karger.com/doi/10.1159/000516648).
Participating sites were provided 2-3 TcB devices which were calibrated daily as per manufacturer's instructions. As per hospital policy, with every medically indicated TSB measurement, a single $\mathrm{TcB}$ (average of 3 measurements) was concomitantly taken at both the forehead and sternum by nurses from time of parental consent up to 10 days, in the NICU. TcB sampling was repeated in each infant at every subsequently required TSB. Phototherapy was started as medically indicated based on TSB measurements. During phototherapy, phototherapy lights were turned off when TSB and $\mathrm{TcB}$ measurements were taken at each site. Nursing staff at all 3 sites were trained by the primary site investigator on how to perform TcB measurements as per procedures described above. Research assistants then collected the results directly from the meter and entered them on the data collection form.

Also recorded were infant age (in hours) at each TSB-TcB measurement and the time of initiation of phototherapy. Maternal and infant demographic data and clinical information were collected via standardized chart extraction.

\section{Data Analyses}

The primary study outcome was the agreement between $\mathrm{TcB}$ and TSB. Agreement was assessed among all infants ("overall"), before and after initiation of phototherapy, by anatomical site (forehead vs. sternum), and by infant ethnicity, determined by maternal self-identified ethnicity (Canadian Caucasian, Southeast Asian, South Asian, and African or Caribbean). To express the level of agreement between TcB and TSB measurements, BlandAltman plots and Lin's concordance correlation coefficient (CCC) were generated. Adapted Bland-Altman plots were weighted for multiple TcB and TSB measurements per infant and the 2 sites of TcB measurement (forehead and sternum), with 95\% LOA [15, 16].

Agreement was assessed, overall, in all preterm infants and further stratified by gestational age (24-28, 29-32, and 33-35 weeks' gestation at birth). Bland-Altman plots were also developed for TcB-TSB measurement differences before or after phototherapy, anatomical site of measurement (forehead vs. sternum), and infant ethnicity.

To assess the use of $\mathrm{TcB}$ as a potential screening test for hyperbilirubinemia, sensitivity, specificity, positive predictive value (PPV), negative predictive value (NPV), and positive and negative likelihood ratios were calculated at clinically important TSB cut points as recommended by Maisels et al. [5] and which are widely used in North America. For preterm infants born at 24-28 weeks, the lack of $\mathrm{TcB}$ measurements prior to phototherapy prohibited any similar analysis, as described below. The recommended TSB cut points are 103-171 $\mu \mathrm{mol} / \mathrm{L}$ in infants born at 29-32 weeks and 171-205 $\mu \mathrm{mol} / \mathrm{L}$ in infants at 33-35 weeks $[1,5]$. Accordingly, a rounded off cut point TSB $>100 \mu \mathrm{mol} / \mathrm{L}$ was applied to infants born at 29-32 weeks' gestation, and a rounded off cut point $>170$ $\mu \mathrm{mol} / \mathrm{L}$ was used for those at 33-35 weeks. Receiver operating characteristic curves, and the respective area under the curves (AUC), were also calculated. Finally, TcB cut points were determined with at least $90 \%$ sensitivity to detect a TSB of $>100 \mu \mathrm{mol} / \mathrm{L}$ at 29-32 weeks' gestation and a TSB of $>170 \mu \mathrm{mol} / \mathrm{L}$ at $33-35$ weeks.

\section{Sample Size Calculation}

In one previous study, the mean TSB was $146 \mu \mathrm{mol} / \mathrm{L}$, with a standard deviation of the average difference between TSB and TcB 
Table 1. Characteristics of the 296 preterm infants included in the study

\begin{tabular}{lc}
\hline Characteristic & Measurement \\
\hline Median (IQR) gestational age, weeks & $31.0(28.0-33.0)$ \\
Mean (SD) birth weight, g & $1,558.8(612.8)$ \\
Sex (female) & $134(45.3)$ \\
\hline Mode of delivery & $180(60.8)$ \\
Cesarean & $101(34.1)$ \\
Vaginal & $15(5.1)$ \\
Unknown & $110(37.2)$ \\
\hline Maternal ethnicity & $90(30.4)$ \\
Canadian Caucasian & $27(9.1)$ \\
Unknown & $26(8.8)$ \\
Southeast Asian & $20(6.7)$ \\
South Asian & $12(4.1)$ \\
African or Caribbean & $6(2.0)$ \\
Hispanic & $5(1.7)$ \\
Middle Eastern & $42.9(30.9)$ \\
First Nations or Inuit & 172 \\
Mean (SD) number of hours from birth to initiation of phototherapy & 684 \\
TcB measurements among all infants, prior to initiation of phototherapy, $N$ & 138 \\
TcB measurements among all infants, after initiation of phototherapy, $N$ & 34 \\
TSB measurements $>100 \mu$ mol/L prior to the initiation of phototherapy, $n$ & \\
TSB measurements $>170 \mu$ mol/L prior to the initiation of phototherapy, $n$ & \\
\hline
\end{tabular}

All data are shown as $n(\%)$ unless otherwise indicated. TcB, transcutaneous bilirubin; TSB, total serum bilirubin.

measurements of $30 \mu \mathrm{mol} / \mathrm{L}$ and negligible variation by gestational age or ethnicity [17]. With our a priori sample size calculation of a minimum of 271 infants, the current study sample of 296 infants was sufficient to detect a minimum TcB-TSB difference of $4 \mu \mathrm{mol} / \mathrm{L}$, at a conventional 2 -sided $p$ value of 0.05 and a statistical power of $80 \%$. Analyses were conducted using NCSS 12 and SPSS 26.

\section{Results}

Out of 344 preterm infants born at $24^{0 / 7}$ and $35^{6 / 7}$ weeks, 296 preterm infants received at least $1 \mathrm{TcB}$ measurement (online suppl. Fig. 1). The median gestational age at birth was 31.0 weeks (IQR 28.0-33.0) among an ethnically diverse group of mothers (Table 1). Each of the 296 infants received a mean (SD) of 7.0 (3.6) TSB measurements. There were 856 paired TcB and TSB measurements done at both the forehead and sternum, with a mean of 3.0 (1.9) paired measurements per infant performed at a median age of $105 \mathrm{~h}$ (IQR 68-151).

Among all 296 infants, the overall mean TcB-TSB difference was $-24.5 \mu \mathrm{mol} / \mathrm{L}$ (to convert TSB to $\mathrm{mg} / \mathrm{dL}$ divide by 17.1 ) (95\% LOA -103.3 to 54.3 ) (Fig. 1a). There were 29 (3.4\%) measures above the upper LOA and 73 (8.5\%) below the lower LOA, with no visual evidence of any greater deviation at higher mean bilirubin concentrations (Fig. 1a). Furthermore, TcB underestimated TSB in 650 of all measures $(75.9 \%)$ and overestimated TSB in 206 of all measures (24.1\%) (Fig. 1a).

\section{TcB Measurement before and after Phototherapy, All \\ Preterm Infants}

In total, 252 neonates (85.1\%) received phototherapy, at a median of $29.0 \mathrm{~h}$ of age (IQR 26.0-55.0). A total of 172 paired measurements were done prior to phototherapy and 684 following phototherapy. Among 79 infants born at 24-28 weeks, 229 out of 241 measurements (95.0\%) were done after initiation of phototherapy, with 76 infants (96.2\%) receiving phototherapy by a median of $26.0 \mathrm{~h}$ of age (IQR 15.0-29.0).

Among all newborns, the overall mean TcB-TSB difference at the combined forehead and sternum was much smaller prior to the initiation of phototherapy (1.6 $\mu \mathrm{mol} / \mathrm{L}, 95 \% \mathrm{LOA}-73.4$ to 76.5 ) (Fig. 1b) than after $(-31.1 \mu \mathrm{mol} / \mathrm{L}, 95 \% \mathrm{LOA}-105.5$ to 43.4$)$ (Fig. 1c). TcB overestimated TSB in $99(57.6 \%)$ measures prior to the 


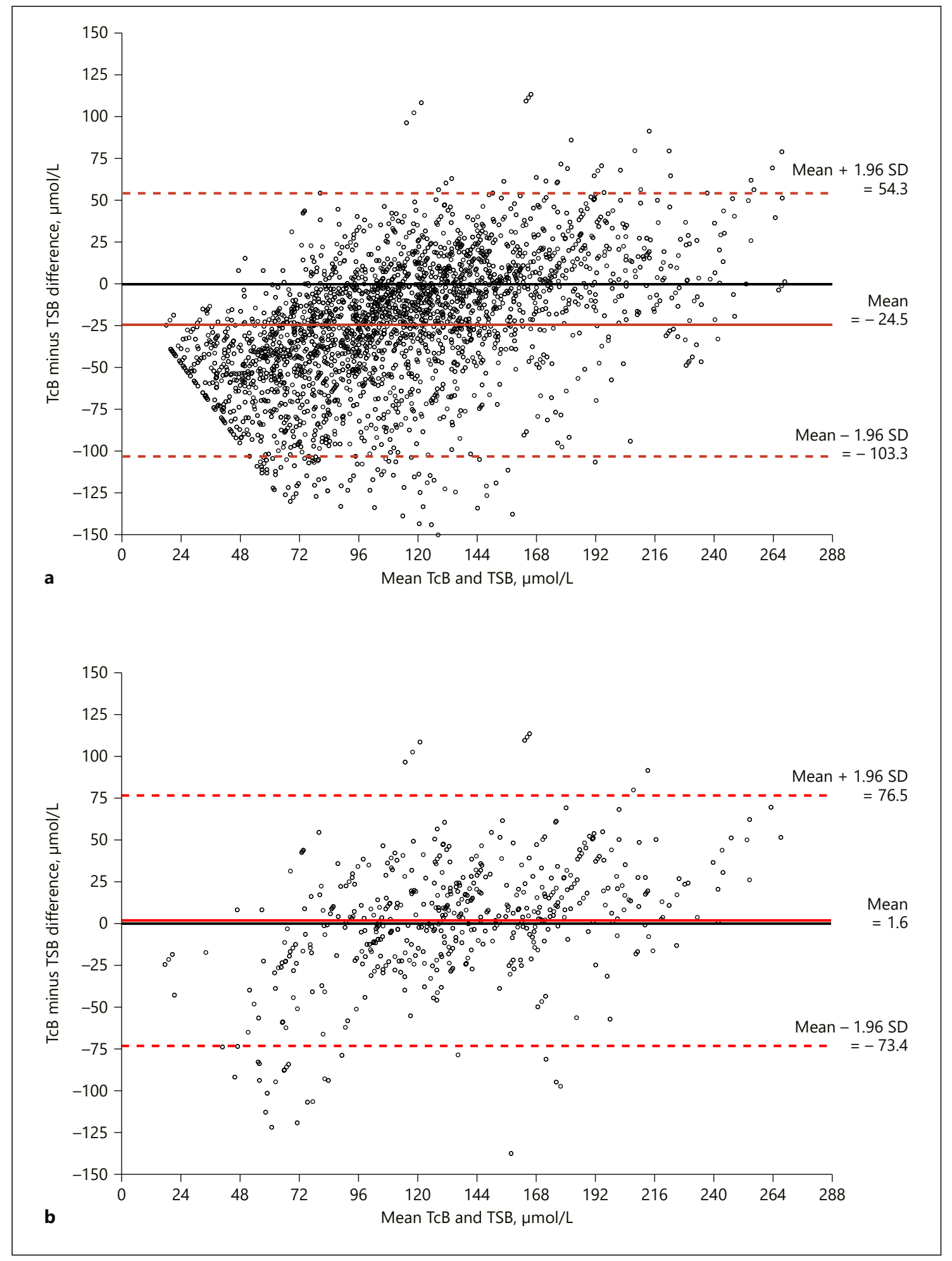

Fig. 1. Bland-Altman plots of paired TcB and TSB measurements among all 296 preterm infants at 24-35 weeks' gestation, measured overall (a), prior to (b), and after (c) initiation of phototherapy. TcB, transcutaneous bilirubin; TSB, total serum bilirubin.

(Figure continued on next page.) 


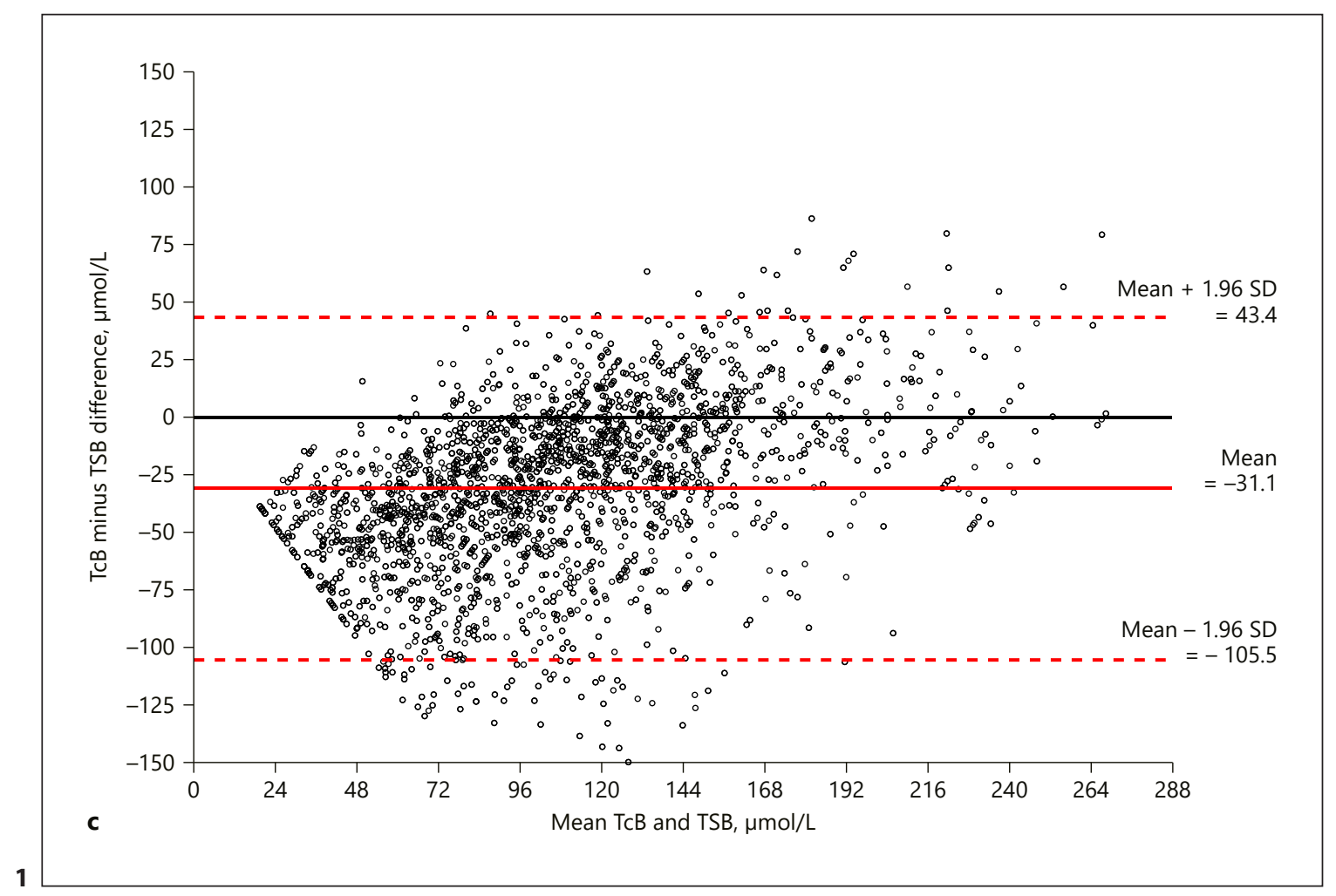

start of phototherapy and underestimated TSB in 577 (84.4\%) measures after. The corresponding Lin's CCC was 0.76 (95\% CI: 0.69-0.81) prior to starting phototherapy and 0.64 (95\% CI: 0.60-0.67) after.

\section{Sites of TcB Measurement}

The overall mean TcB-TSB difference at the forehead $(-15.2 \mu \mathrm{mol} / \mathrm{L}, 95 \% \mathrm{LOA}-86.8$ to 56.3$)$ was less pronounced than at the sternum $(-24.4 \mu \mathrm{mol} / \mathrm{L}, 95 \% \mathrm{LOA}$ -112.9 to 64.0 ) (Table 2). This was consistent after receipt of phototherapy and upon stratifying by gestational age at birth (Table 2). Lin's CCC between TSB and TcB at the forehead was consistently higher than at the sternum (Table 2).

\section{TcB Measurement by Gestational Age Groups}

Regardless of phototherapy, 241 paired TcB-TSB measurements were obtained in 79 neonates born at 24-28 weeks, 381 paired measurements in 119 infants born at 29-32 weeks, and 234 paired measurements in 98 infants born at 33-35 weeks. At the forehead and sternal sites combined, the mean TcB-TSB difference was -31.4 $\mu \mathrm{mol} / \mathrm{L}(95 \% \mathrm{LOA}-95.3$ to 32.4$)$ at $24-28$ weeks, -25.5 $\mu \mathrm{mol} / \mathrm{L}(95 \% \mathrm{LOA}-102.7$ to 51.8$)$ at $29-32$ weeks, and
$-15.9 \mu \mathrm{mol} / \mathrm{L}(95 \% \mathrm{LOA}-107.4$ to 75.6$)$ at $33-35$ weeks. However, upon limiting to $\mathrm{TcB}$ at the forehead (where higher overall LOA were observed regardless of phototherapy), the mean TcB-TSB difference was less pronounced, especially among late-preterm infants (Fig. 2c). This was consistently seen when stratified by receipt of phototherapy and site of measurement (Table 2). TcB underestimated TSB in $216(89.6 \%)$ of the measures done in infants born at 24-28 weeks, $302(79.3 \%)$ of those at 2932 weeks, and 132 (56.4\%) of the measures in infants born at 33-35 weeks.

\section{Influence of Ethnicity on $T c B$}

The overall LOA between TSB and TcB (both prior to and after the initiation of phototherapy) were similar across the 4 main ethnic groups, including infants born to a mother of African or Caribbean ethnicity (online suppl. Fig. 2).

\section{Performance of TcB at Predefined TSB Levels}

Among infants born at 29-32 weeks' gestation and at the recommended TSB cut point of $>100 \mu \mathrm{mol} / \mathrm{L}$, prior to phototherapy, forehead $\mathrm{TcB}$ had an AUC of 0.76 (95\% CI: 0.59-0.87) (Fig. 3a; Table 3,). A forehead TcB cut point of 
Table 2. Agreement between measurement of TcB and TSB among all 296 preterm infants included in the study

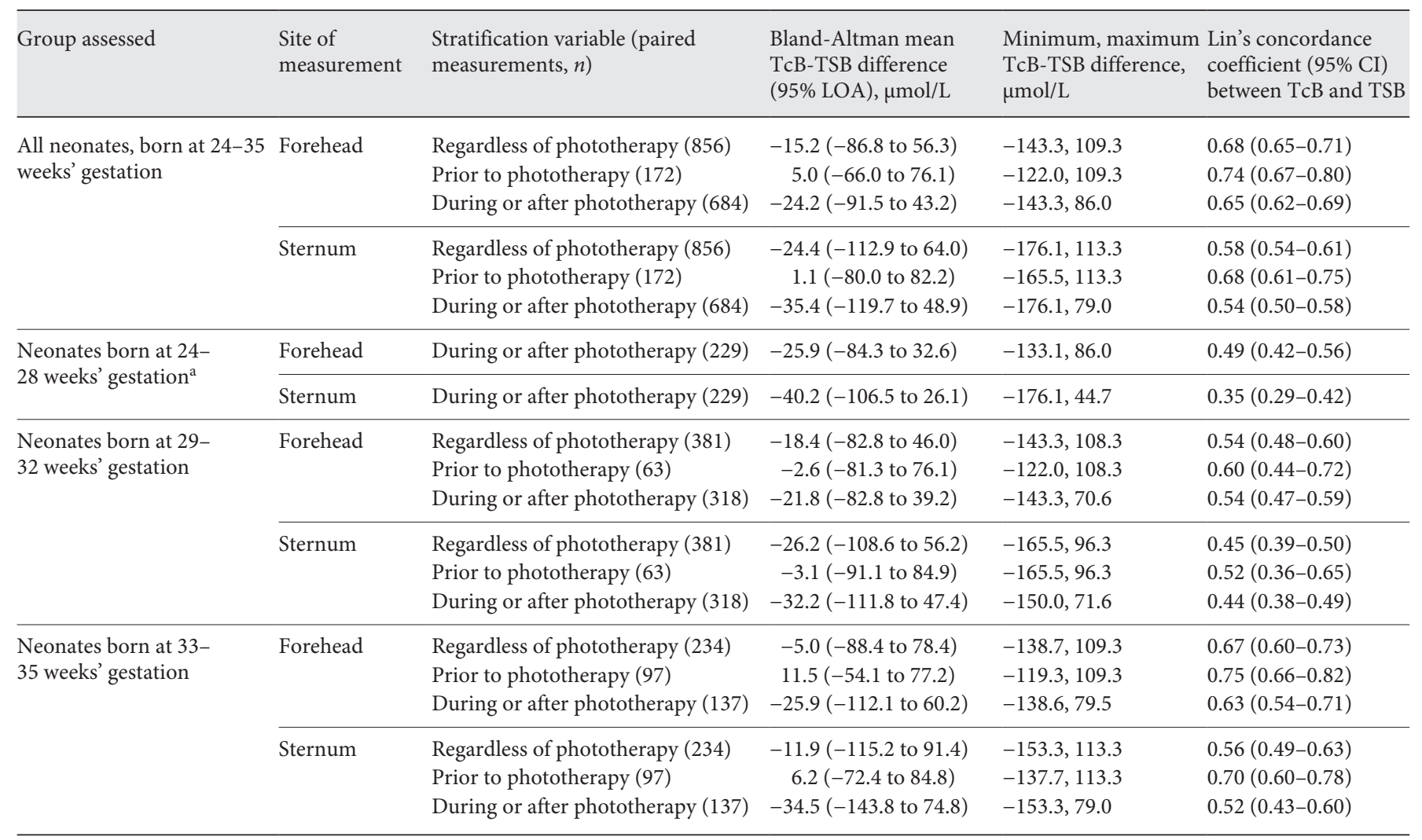

Shown are measures of agreement stratified by anatomical site of TcB assessment, gestational age at birth, and receipt of phototherapy. TcB, transcutaneous bilirubin; TSB, total serum bilirubin; LOA, limits of agreement. ${ }^{a}$ Only 11 neonates born at $24-28$ weeks' gestation had the opportunity to have TSB or TcB measured before initiation of phototherapy, providing 12 paired TcB measurements. Accordingly, the related data are only presented for those infants receiving phototherapy.

Table 3. Test characteristics of TcB measurement at recommended TSB cut points

\begin{tabular}{|c|c|c|c|c|c|c|c|}
\hline Neonates born at $33-35$ weeks' gestation & $>170$ & $83(64-94)$ & $78(66-87)$ & $61(45-77)$ & $91(81-97)$ & $3.7(2.3-6.0)$ & $0.22(0.10-0.50)$ \\
\hline
\end{tabular}

$\mathrm{TcB}$, transcutaneous bilirubin; TSB, total serum bilirubin; PPV, positive predictive value; NPV, negative predictive value.

$85 \mu \mathrm{mol} / \mathrm{L}$ had a sensitivity of $92 \%$ (95\% CI: 80-98), specificity 60\% (95\% CI: 32-84), PPV 88\% (95\% CI: 76-95), NPV 69\% (95\% CI: 38-91), and respective positive and negative likelihood ratios of 2.3 (95\% CI: $1.2-4.3$ ) and 0.14 (95\% CI: 0.05-0.39) to detect the recommended TSB threshold of $>100 \mu \mathrm{mol} / \mathrm{L}$ at 29-32 weeks' gestation.

In preterm infants born at 33-35 weeks' gestation and at the recommended TSB cut point of $>170 \mu \mathrm{mol} / \mathrm{L}$, prior to phototherapy, forehead TcB had an AUC of 0.86 (95\%
CI: 0.76-0.92) (Fig. 3b; Table 3, ). A forehead TcB cut point of $156 \mu \mathrm{mol} / \mathrm{L}$ had a sensitivity of $90 \%$ (95\% CI: 73-98), specificity 69\% (95\% CI: 57-80.0), PPV 55\% (95\% CI: 40-70), NPV 94\% (95\% CI: 83-99), and respective positive and negative likelihood ratios of 2.9 (95\% CI: 2.0-4.2) and 0.15 (95\% CI: $0.05-0.44)$ to detect the recommended TSB threshold of $>170 \mu \mathrm{mol} / \mathrm{L}$ among infants born at 33-35 weeks. 


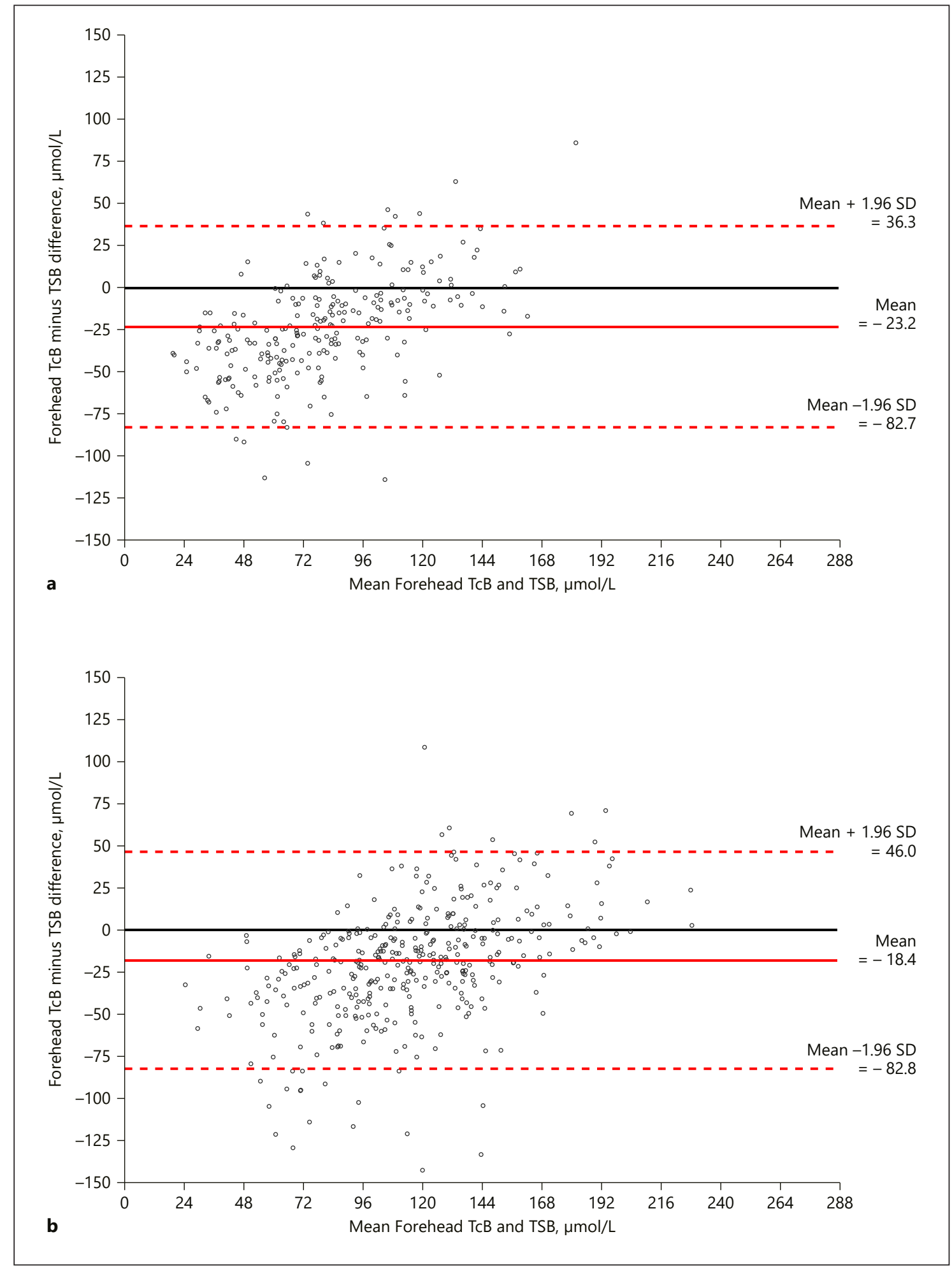

Fig. 2. Bland-Altman plot of paired forehead TcB and TSB measurements among neonates born at 24-28 weeks' gestation (a), 29-32 weeks' gestation (b), and 33-35 weeks' gestation (c). TcB, transcutaneous bilirubin; TSB, total serum bilirubin.

(Figure continued on next page.) 


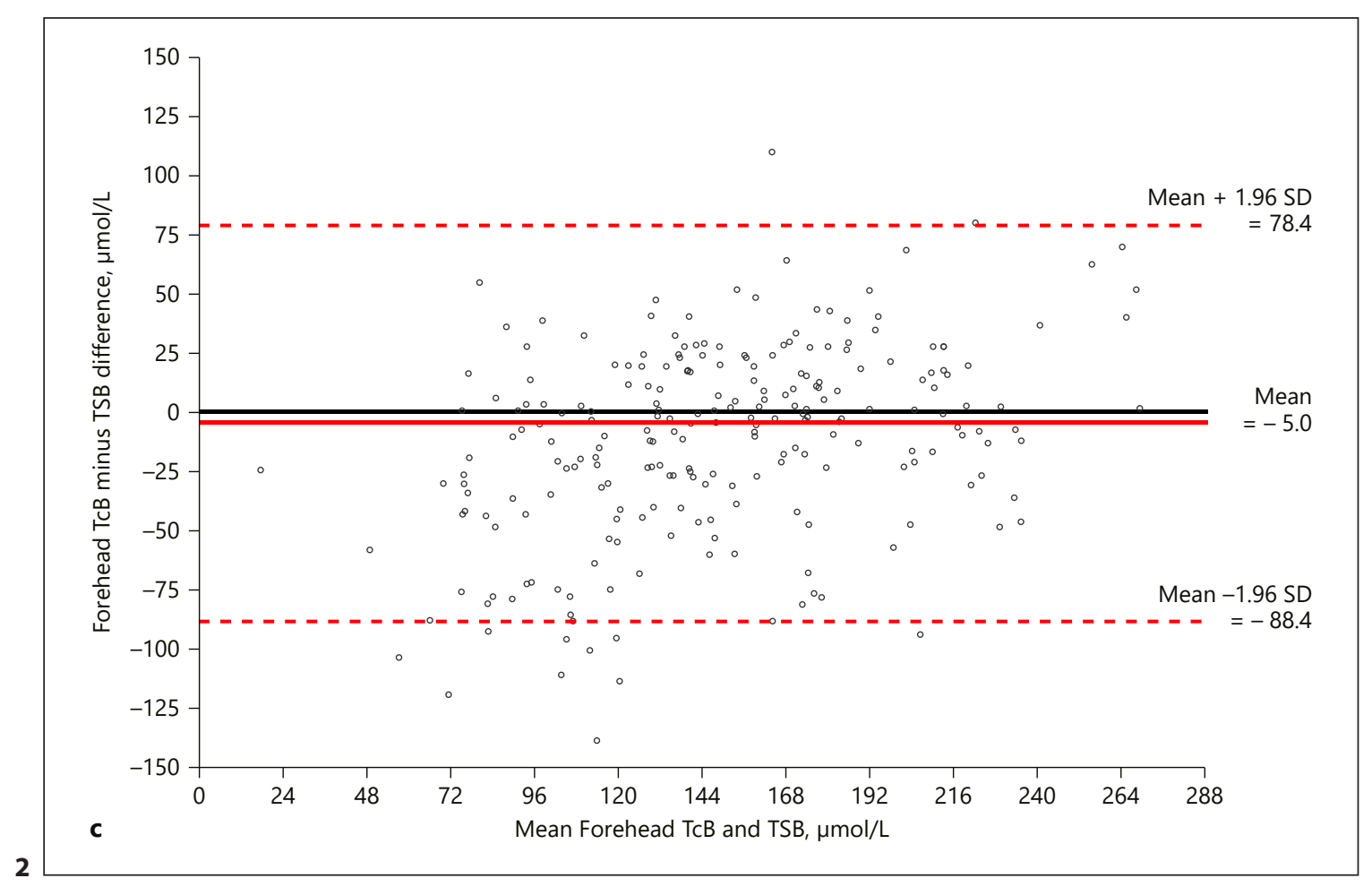

\section{Discussion}

In this multisite, prospective cohort study of Canadian infants born preterm at $24^{0 / 7}$ to $35^{6 / 7}$ weeks' gestation, $\mathrm{TcB}$ measured with the JM-105 at the forehead and sternum offered a reasonably accurate assessment of TSB, especially prior to starting phototherapy in preterm infants born between 33 and 35 weeks' gestation. In preterm infants born at 33-35 weeks' gestation, TcB demonstrated better agreement with TSB than among those born before 33 weeks' gestation. Maternal ethnicity did not appreciably affect TcB.

As per our primary objective, we studied the agreement between TcB and TSB measurements among preterm infants using multiple $\mathrm{TcB}$ measurements per infant. Specifically, this study assessed the impact of phototherapy initiation, the anatomical site of measurement, gestational age at birth, and maternal ethnicity. This study used an adapted version of the Bland-Altman analysis, which accounts for repeated bilirubin measurements in the same infant $[15,16]$. Previously, a regression analysis was employed to account for multiple measurements per infant [18]. The inclusion of a large number of repeated samples permitted a comprehensive analysis of $\mathrm{TcB}$ measurements within a diverse sample of neonates, surpassing the sample size from a previously completed study in India using the BiliChek [19].

Similar to previous studies of $\mathrm{TcB}$ among preterm infants, our study reported a mean TcB-TSB difference of $\leq 26 \mu \mathrm{mol} / \mathrm{L}[9,18,19]$. After initiation of phototherapy and at a greater degree of prematurity, TcB-TSB agreement worsened. This is consistent with previous research that showed greater agreement between $\mathrm{TcB}$ and TSB prior to initiating phototherapy using the JM103 and JM-105 devices [12, 20, 21]. As in prior studies, we saw no appreciable difference whether $\mathrm{TcB}$ was measured at the forehead or sternum prior to phototherapy $[11,14]$; however, after the initiation of phototherapy, the current study suggests that the forehead may be the more preferred site for $\mathrm{TcB}$ measurement when using the JM-105 device. One reason for this may be the reduced exposure to phototherapy at the forehead from the eye mask used during treatment [22]. However, TSB measurements should be used to make clinical decisions, especially after the initiation of phototherapy. As with former studies $[17,23]$, ethnicity does not appear to appreciably affect the agreement between TcB and TSB measurements.

As a limitation, fewer TcB measurements were available before initiation of phototherapy than after, espe- 


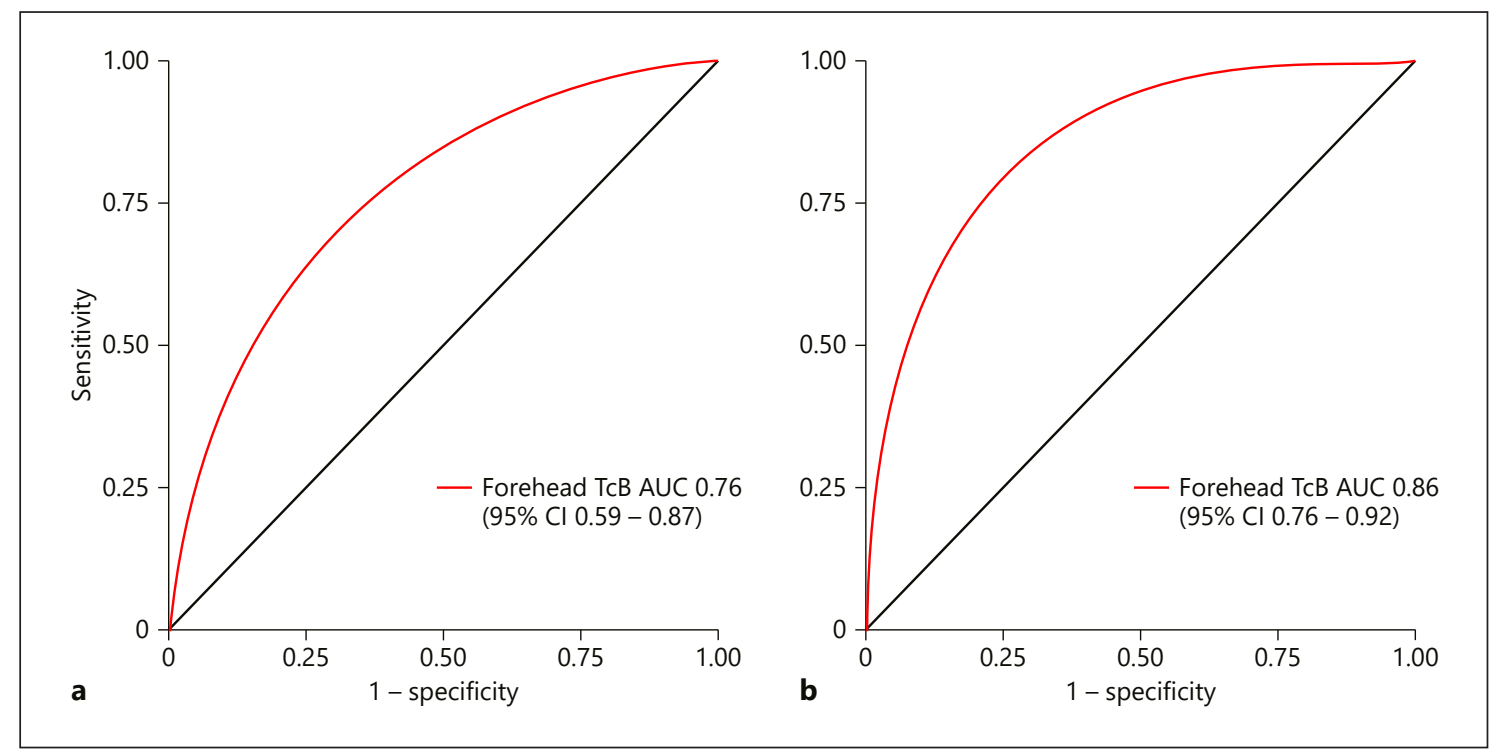

Fig. 3. Receiver operating curves for $\mathrm{TcB}$ measured at the forehead at the recommended TSB thresholds of $>100$ $\mu \mathrm{mol} / \mathrm{L}$ at 29-32 weeks' gestation (a) and $>170 \mu \mathrm{mol} / \mathrm{L}$ at 33-35 weeks' gestation (b). TcB, transcutaneous bilirubin; TSB, total serum bilirubin.

cially among infants born at $24-28$ weeks' gestation who started on phototherapy at a median of $26 \mathrm{~h}$ of age. The recruitment of extremely preterm infants was challenging in terms of obtaining parental consent prior to initiation of phototherapy. This study did not assess TcB-TSB agreement in relation to the time since phototherapy cessation and therefore did not differentiate between during or after phototherapy. Finally, as this study used the JM105 device, the current findings may not be reflective of the performance of other $\mathrm{TcB}$ devices.

The clinical utility of $\mathrm{TcB}$ as a screening tool was also assessed at specific recommended treatment TSB thresholds in 2 preterm infant groups. Among infants born at 29-32 weeks, forehead TcB had an AUC of 0.76 to detect the recommended TSB threshold of $>100 \mu \mathrm{mol} / \mathrm{L}$. At this gestational age, a forehead TcB cut point of $85 \mu \mathrm{mol} / \mathrm{L}$ had the necessary sensitivity of $92 \%$ to detect the recommended TSB threshold of $>100 \mu \mathrm{mol} / \mathrm{L}$, even though the specificity was only $60 \%$. In preterm infants at 33-35 weeks' gestation, the corresponding AUC was 0.86 to detect the recommended TSB threshold of $>170 \mu \mathrm{mol} / \mathrm{L}$. Among these infants, a forehead $\mathrm{TcB}$ cut point of 156 $\mu \mathrm{mol} / \mathrm{L}$ had the requisite sensitivity of $90 \%$, with a slightly better specificity of $69 \%$ to detect the recommended TSB threshold of $>170 \mu \mathrm{mol} / \mathrm{L}$. Accordingly, TcB may be useful in screening for hyperbilirubinemia in low-risk preterm infants born at 33-35 weeks prior to the initia- tion of phototherapy. Among preterm infants born at 2932 weeks' gestation, TcB should be used more carefully as TSB approaches treatment thresholds. In all preterm infants, the recommended TSB thresholds of treatment need to be considered when deciding to use $\mathrm{TcB}$ in this population.

Clinicians should remain cognizant of the treatment thresholds for hyperbilirubinemia, based on gestational age at birth and other clinical factors for developing bilirubin toxicity [5]. Once an infant is on phototherapy, blood testing is often repeated regularly, to monitor the effect of phototherapy on bilirubin levels [12]. The tendency of TcB to underestimate TSB after the initiation of phototherapy in $84 \%$ of $\mathrm{TcB}$ measurements after the initiation of phototherapy seems problematic: relying on $\mathrm{TcB}$ alone might lead to improper discontinuation of phototherapy [12]. As such, TSB measurements should be used to make clinical decisions after the initiation of phototherapy, especially since most preterm infants start phototherapy after the first or second day of life. As a potential solution to the underperformance of $\mathrm{TcB}$ after initiation of phototherapy, for example, some small studies in term and preterm infants explored the effectiveness of measuring TcB on covered skin $[20,24]$. A similar approach, with a larger sample size, should be considered in preterm infants, to determine the effectiveness of TcB after phototherapy. 
In addition to procedural pain, repeated blood sampling in preterm infants can contribute to anemia [25]. Approximately $0.5 \mathrm{~mL}$ of blood sampling is required for TSB measurement in late-preterm infants. In the current study, each newborn received a mean of 7 TSB measurements, amounting to $3.5 \mathrm{~mL}$ of cumulative blood loss. The careful use of $\mathrm{TcB}$ as a screening tool may reduce blood sampling, especially in mid- and late-preterm infants.

\section{Conclusion}

Among preterm infants born at 33-35 weeks' gestation, TcB with the JM-105 may offer a noninvasive, immediate approach to screening for hyperbilirubinemia prior to phototherapy. Careful use of TcB should be considered in infants born at $<33$ weeks' gestation when TSB levels are approaching phototherapy thresholds. Its notable underestimation of TSB measurements after the initiation of phototherapy warrants limited use after the initiation of phototherapy and use of TSB measurements for clinical decision-making.

\section{Acknowledgment}

Some members of the Neonatal Hyperbilirubinemia Consortium: Rachael Allen, Jeffrey Antwi, Jillian Baker, Shangari Baleswaran, Matthew Barozzino, Tony Barozzino, Maria Casalino, Sohail Cheema, Mary Debono, Maria Ghobrial, Aasha Gnanalingam, Aaditeya Jhaveri, Sharmilaa Kandasamy, Melissa Librach, Saisujani Rasiah, Sureka Selvakumaran, Heet Sheth, Ashvinie Sritharan, Geoffrey Travis, Jennifer Twiss, and Gayathri Visvanathaiyer.

\section{Statement of Ethics}

Institutional Research Ethics Board approval was obtained at all 3 participating sites, and informed written consent was provided by the parent(s) (St. Michael's Hospital REB reference \#15-376).

\section{Conflict of Interest Statement}

The authors have no conflicts of interest to declare.

\section{Funding Sources}

This study was funded by Drager Medical Systems Inc., Germany, and the University of Toronto Open Fellowship Award, Toronto, Canada.

\section{Author Contributions}

Thivia Jegathesan, Dr. Joel Ray, and Dr. Michael Sgro* conceptualized the design of the study, drafted the initial manuscript, and reviewed and revised the manuscript. Dr. Howard Berger and Dr. Robin Hayeems assisted in the design of the study, assisted with the analyses, and critically reviewed and revised the manuscript. Dr. Vibhuti Shah and Dr. Douglas Campbell assisted in the design of the study, coordinated and supervised data collection, and reviewed and revised the manuscript.

\section{Study Registration}

This study is registered on https://clinicaltrials.gov/ct2/show/ NCT02774434.

\section{References}

1 American Academy of Pediatrics. Management of hyperbilirubinemia in the newborn infant 35 or more weeks of gestation. Pediatrics. 2004;114(1):297-316.

2 Barrington K, Sankaran K; Canadian Pediatric Society. Guidelines for detection, management and prevention of hyperbilirubinemia in term and late preterm newborn infants $(35$ or more weeks' gestation) - summary. J Paediatr Child Health. 2007;12(5):401-18.

3 Sgro M, Kandasamy S, Shah V, Ofner M, Campbell D. Severe neonatal hyperbilirubinemia decreased after the 2007 canadian guidelines. J Pediatr. 2016;171:43-7.

4 Bhutani VK, Wong RJ. Bilirubin neurotoxicity in preterm infants: risk and prevention. J Clin Neonatol. 2013;2(2):61-9.
5 Maisels MJ, Watchko JF, Bhutani VK, Stevenson DK. An approach to the management of hyperbilirubinemia in the preterm infant less than 35 weeks of gestation. J Perinatol. 2012;32(9):660-4.

6 Hussain AS, Shah MH, Lakhdir M, Ariff S, Demas S, Qaiser F, et al. Effectiveness of transcutaneous bilirubin measurement in managing neonatal jaundice in postnatal ward of a tertiary care hospital in Pakistan. BMJ Paediatr Open. 2017;1(1):e000065.

7 Jnah A, Newberry DM, Eisenbeisz E. Comparison of transcutaneous and serum bilirubin measurements in neonates 30 to 34 weeks' gestation before, during, and after phototherapy. Adv Neonatal Care. 2018;18(2):144-53.
8 Raba AA, O'Sullivan A, Miletin J. Transcutaneous bilirubinometry during and after phototherapy in preterm infants: a prospective observational study. BMJ Paediatr Open. 2020;4(1):e000681.

9 Rohsiswatmo R, Oswari H, Amandito R, Sjakti HA, Windiastuti E, Roeslani RD, et al. Agreement test of transcutaneous bilirubin and bilistick with serum bilirubin in preterm infants receiving phototherapy. BMC Pediatr. 2018;18(1):315.

10 Brummelte S, Grunau RE, Chau V, Poskitt KJ, Brant R, Vinall J, et al. Procedural pain and brain development in premature newborns. Ann Neurol. 2012;71(3):385-96. 
11 Agrawal G, Garg K, Sitaraman S, Sarna A. Comparison of diagnostic accuracy of different sites for transcutaneous bilirubin measurement in early preterm infants. Indian J Pediatr. 2019;86(1):32-7.

12 Hulzebos CV, Vader-van Imhoff DE, Bos AF, Dijk PH. Should transcutaneous bilirubin be measured in preterm infants receiving phototherapy? The relationship between transcutaneous and total serum bilirubin in preterm infants with and without phototherapy. PloS one. 2019;14(6): e0218131.

13 Hynes S, Moore Z, Patton D, O'Connor T, Nugent L. Accuracy of transcutaneous bilirubin versus serum bilirubin measurement in preterm infants receiving phototherapy: a systematic review. Adv Neonatal Care. 2020; 20(6):E118-26.

14 Nagar G, Vandermeer B, Campbell S, Kumar M. Reliability of transcutaneous bilirubin devices in preterm infants: a systematic review. Pediatrics. 2013;132(5):871-81.
15 Bland JM, Altman DG. Agreement between methods of measurement with multiple observations per individual. J Biopharm Stat. 2007;17(4):571-82.

16 Zou GY. Confidence interval estimation for the Bland-Altman limits of agreement with multiple observations per individual. Stat Methods Med Res. 2013;22(6):630-42.

17 Afanetti M, Eleni Dit Trolli S, Yousef N, Jrad I, Mokhtari M. Transcutaneous bilirubinometry is not influenced by term or skin color in neonates. Early Hum Dev. 2014;90(8):41720.

18 Maisels MJ, Coffey MP, Kring E. Transcutaneous bilirubin levels in newborns. J Perinatol. 2015;35(9):739-44.

19 Chawla D, Jain S, Kaur G, Sinhmar V, Guglani $\mathrm{V}$. Accuracy of transcutaneous bilirubin measurement in preterm low-birth-weight neonates. Eur J Pediatr. 2014;173(2):173-9.

20 Costa-Posada U, Concheiro-Guisan A, Taboas-Ledo MF, Gonzalez-Colmenero E, Gonzalez-Duran ML, Suarez-Albo M, et al. Accuracy of transcutaneous bilirubin on covered skin in preterm and term newborns receiving phototherapy using a JM-105 bilirubinometer. J Perinatol. 2019;40:226-31.
21 Castro A, Zozaya C, Cuesta MT, Gonzalez M, Villar G, Alcaraz A. Usefulness of transcutaneous bilirubin assessment measured in nonphoto-exposed skin to guide the length of phototherapy: an observational study. J Perinat Med. 2019;47(5):568-73.

22 Bahr TM, Shakib JH, Stipelman CH, Kawamoto K, Cail K, Lauer S, et al. Improving the bilirubin management program in the newborn nursery: background, aims, and protocol. Neonatology. 2020;117(3):358-64.

23 Ahmed M, Mostafa S, Fisher G, Reynolds TM. Comparison between transcutaneous bilirubinometry and total serum bilirubin measurements in preterm infants. Ann Clin Biochem. 2010;47(Pt 1):72-7.

24 De Luca D, Dell'Orto V. Patched skin bilirubin assay to monitor neonates born extremely preterm undergoing phototherapy. J Pediatr. 2017;188:122-7.

25 Counsilman CE, Heeger LE, Tan R, Bekker V, Zwaginga JJ, te Pas AB, et al. Iatrogenic blood loss in extreme preterm infants due to frequent laboratory tests and procedures. J Matern Fetal Neonatal Med. 2019:1-6. 